\title{
The Anti-Aggressive Effect of Music Therapy in an Animal Model of Schizophrenia
}

\author{
Milad Ahmadi ${ }^{1 \dagger}$, Mehrnaz Banazadeh Dardashti ${ }^{1 \dagger}$, Fariba Karimzadeh ${ }^{1,2^{*}}$
}

${ }^{1}$ Shefa Neuroscience Research Center, Khatam-al-Anbia Hospital, Tehran, Iran.

${ }^{2}$ Cellular and Molecular Research Center, Iran University of Medical Sciences, Tehran, Iran.

\section{A BSTRACT}

I ntroduction: Schizophrenia begins typically in young adulthood (before 25 years old) and about $1 \%$ of people are affected during their lifetime. The antipsychotic drugs have several side effects. This study was aimed to investigate the Mozart's music effect, as a non-invasive treatment, on the schizophrenia. Materials and Methods: Rats aged 21-25 days were divided into four groups: control, music-treated, social isolated (SI) and SI + music-treatment groups. Animals in the control group, without music exposure and music-treated rats, with music exposure, were housed socially. Animals in the SI group, without music exposure, and SI+ music-treatment were housed individually for 6 weeks. Music (Mozart's piano sonata, KV361) was played 24 hours before and during behavioral tests. Social contraction test for each rat was performed. The positive and negative behaviors were scored. Results: The mean number of negative behaviors, such as pursuit, attack, biting and offensive behaviors, in SI and SI + music-treatment increased compared to control and music-treated groups. In addition, biting, up right offensive and treating in SI + music-treatment significantly decreased compared to SI group. In addition, the positive behaviors (walking) in SI + music-treatment group significantly increased compared to SI group. Conclusion: These data suggest the potential of music therapy effects of that listening to music maybe improve the pathological effects of social isolation.

\section{Key words:}

1. Schizophrenia

2. Anxiety

3. Music

4. Social Isolation

* Corresponding Author: Fariba Karimzadeh

E-mail: Fariba_karimzade@yahoo.com

$\dagger$ These authors contributed equally. 


\title{
اثرات ضد برخاشگَىى موسيقى درمانى در مدل حيوانى اسكيزوفرنى
}

\author{
ميلاد احمدى"، مهرناز بنا زاده دردشتى "، فريبا كريم زاده ‘."
}

'مركز تحقيقات علوم اعصاب شفا، بيمارستان خاتم الانبياء، تهران، ايران.

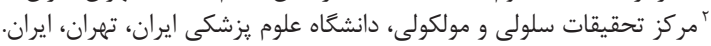

مقدمه: بيمارى اسكيزوفرنيا با شيوع نزديك به يك درصد در جامعه ى جهانى، معمولاً در سنين جوانى

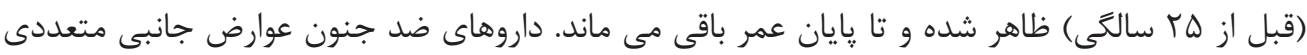

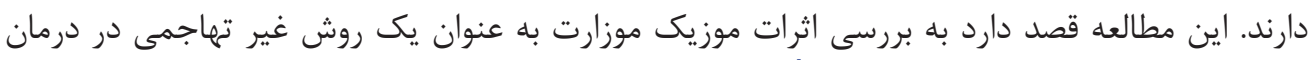

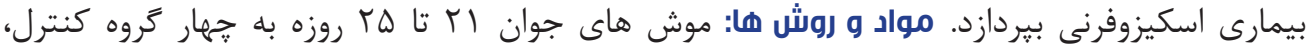

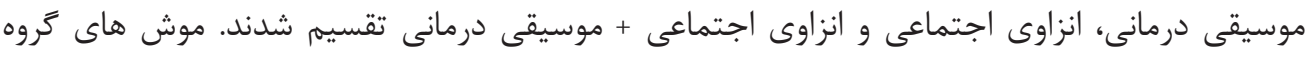

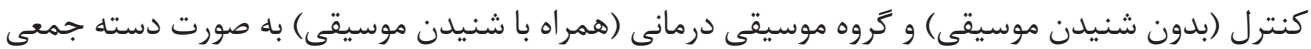

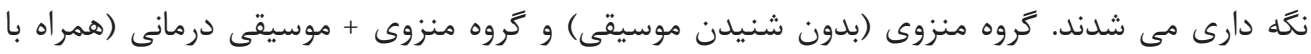

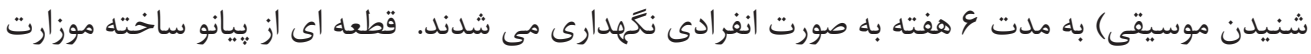

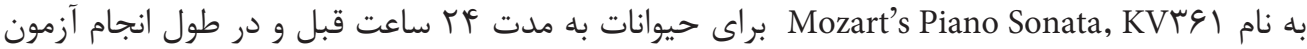

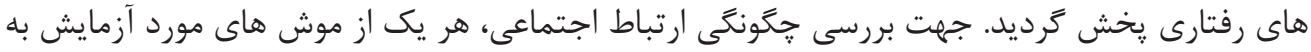

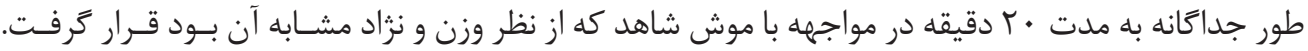

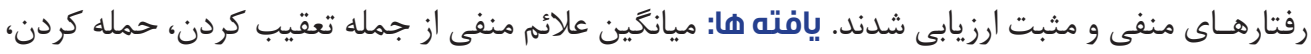

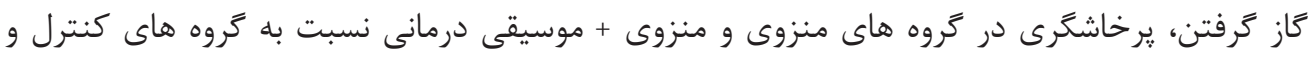

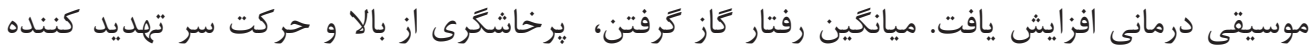

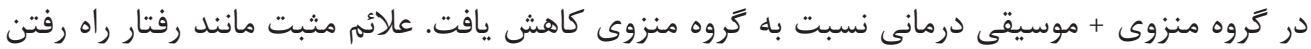

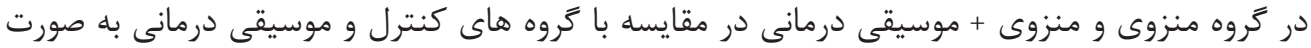

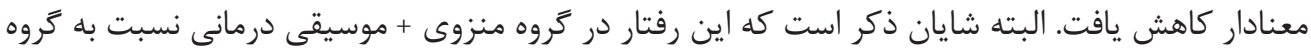

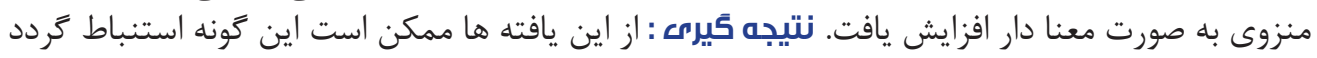

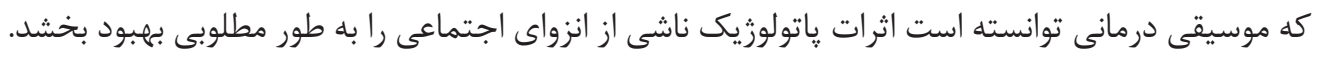




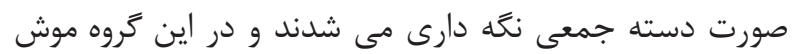

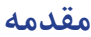

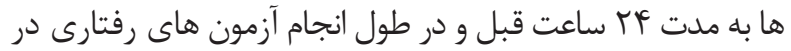

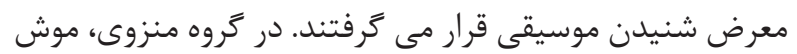

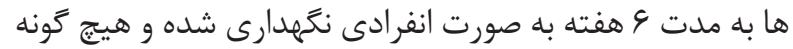

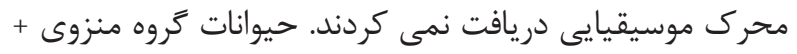

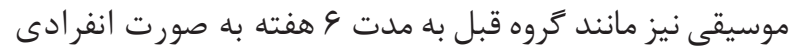

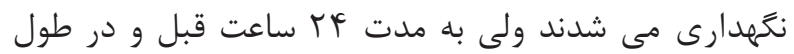

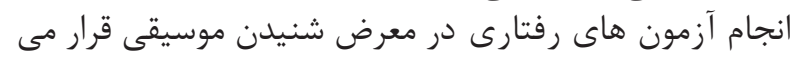

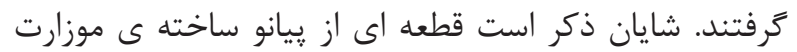

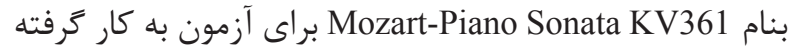

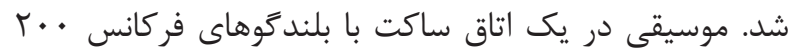

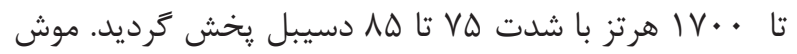

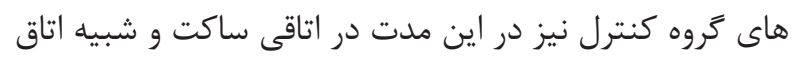

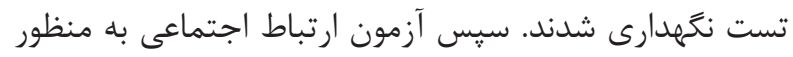

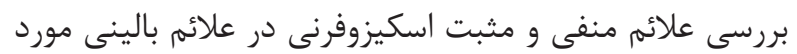
برسى قرار زرفت.

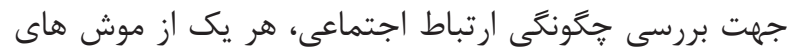

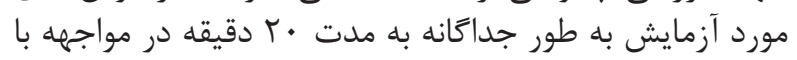

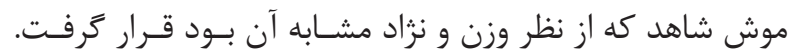

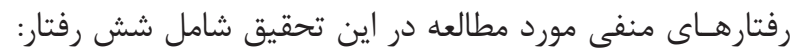

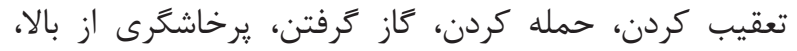

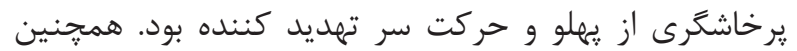

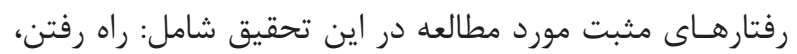

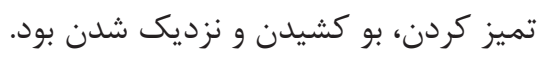
در تمـام مـدت آزمـايش از رفتارهاى موش ها فيلمبردارى

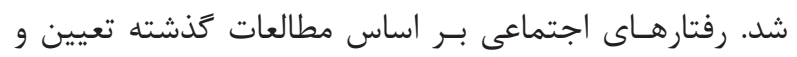

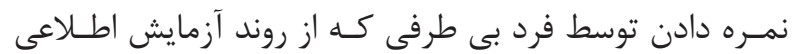

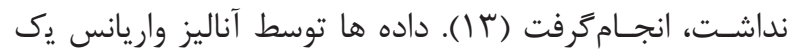

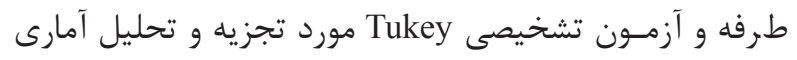

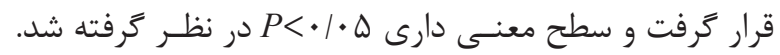

بافتهها

در بررسى علائم منفى، يافته ها حاكى از آن بود كه شنيدن

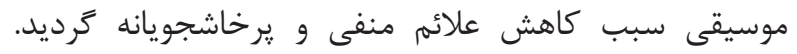

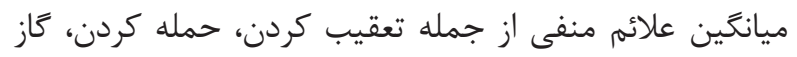

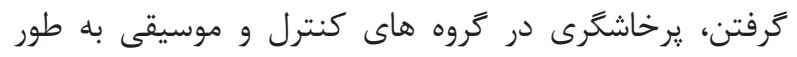

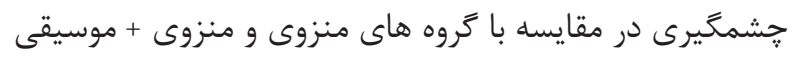

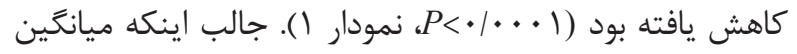

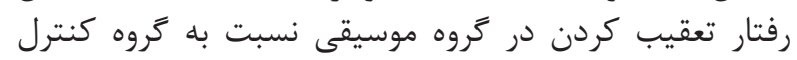

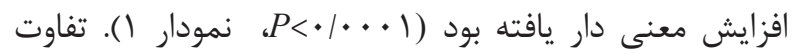

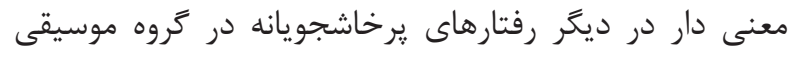

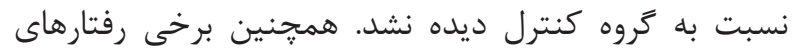

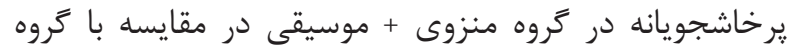

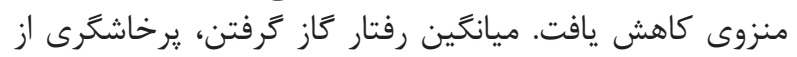

\footnotetext{
${ }^{1}$ Mozart's music

${ }^{2}$ Brain derived neurotrophic factor

${ }^{3}$ Nerve growth factor

${ }^{4}$ Neurogenesis
}

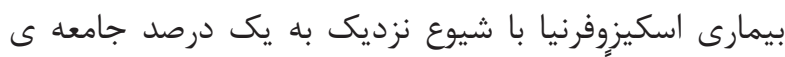

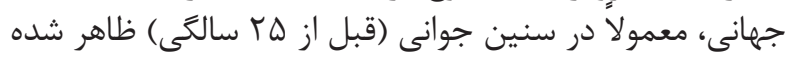

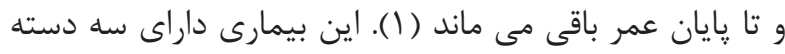

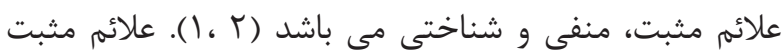

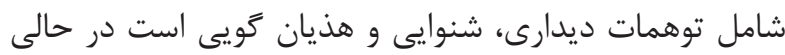

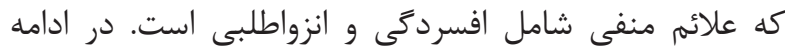

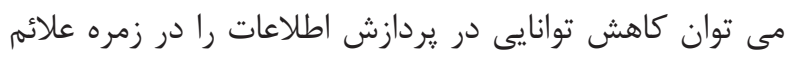

شناختى قرار داد (T).

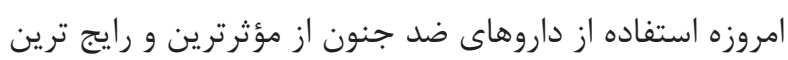

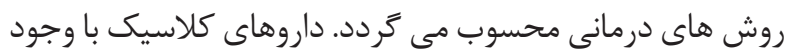

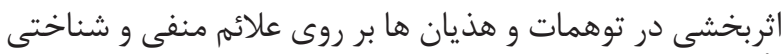

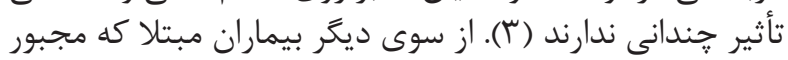

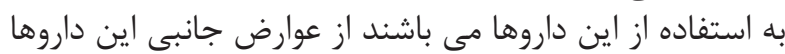

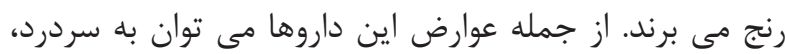

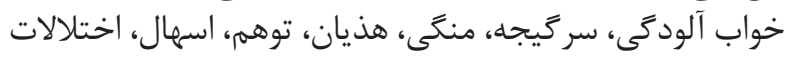

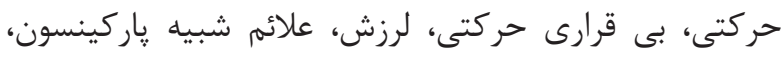

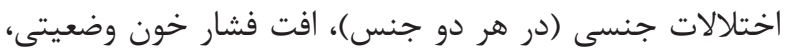

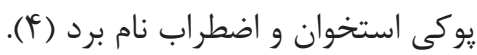

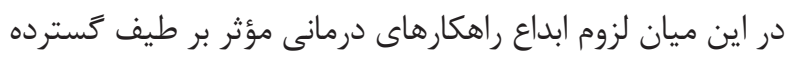

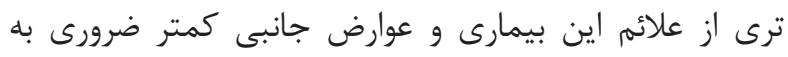

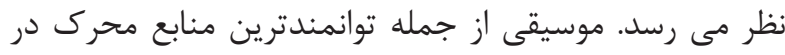

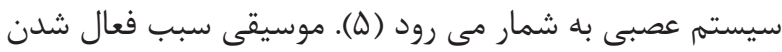

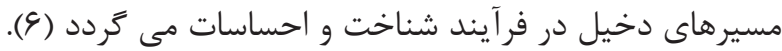

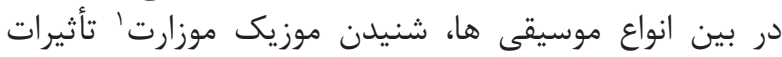

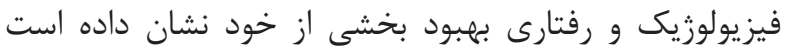
(V، N)

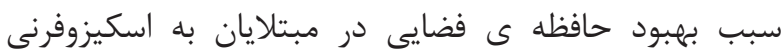

كرديده است (•l. (9).

در موش هايى كه در معرض موسيقى موزارت قرار كرفته بودند

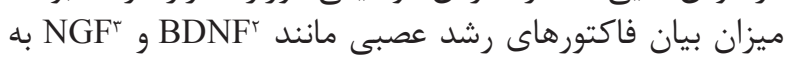

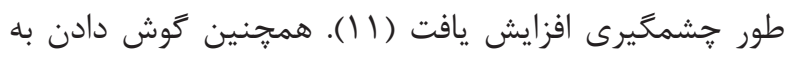

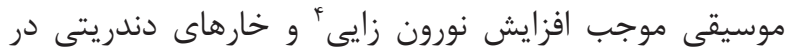

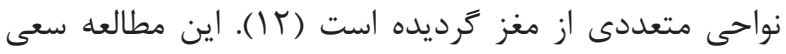

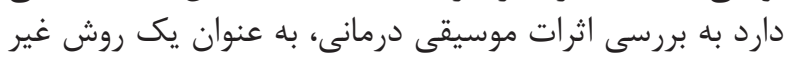

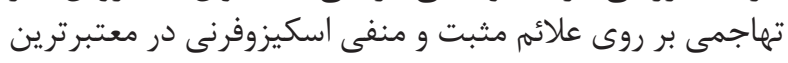

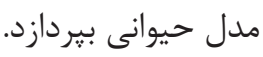

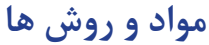

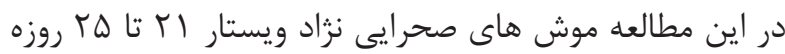

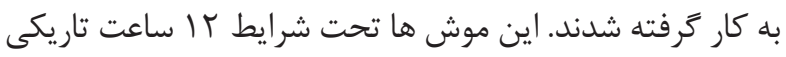

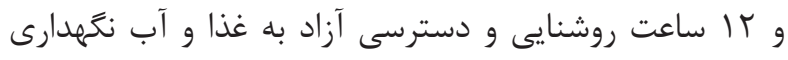

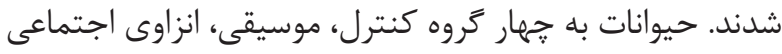

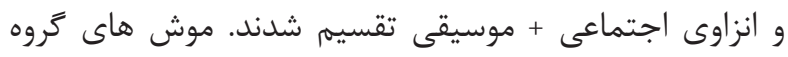

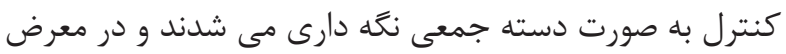

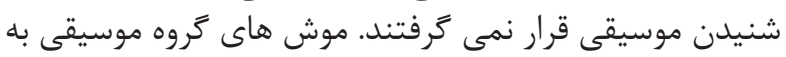




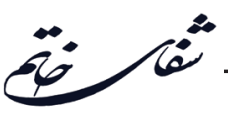

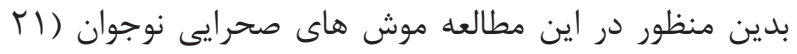

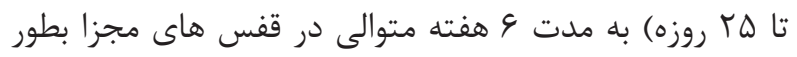

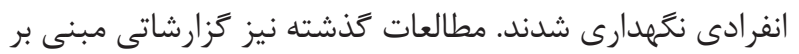

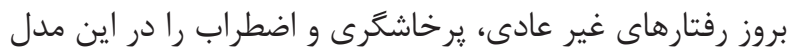

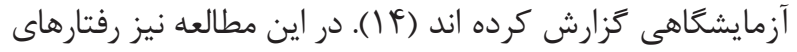

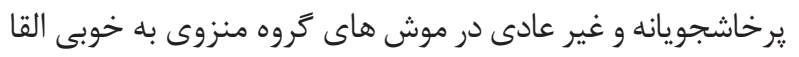

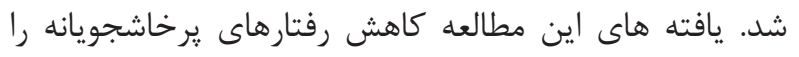

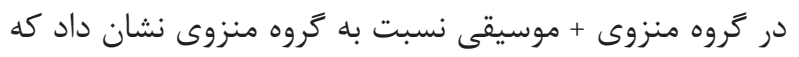

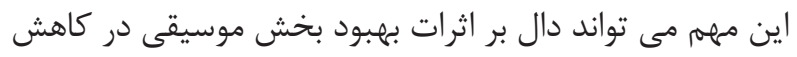
علائم مثبت در اين مدل حيوانى اسكيزوفرنى باشد.

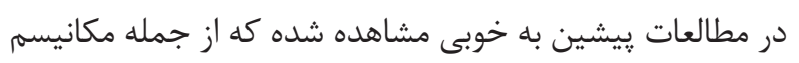

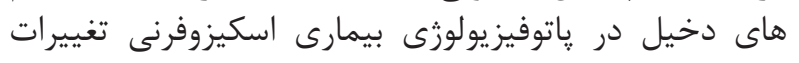

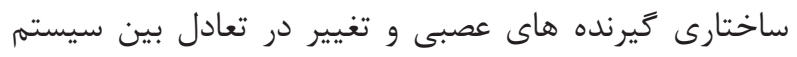

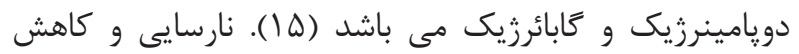

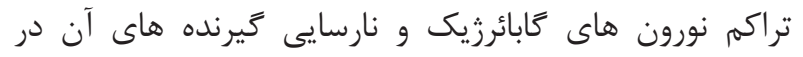

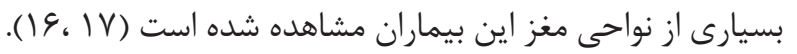

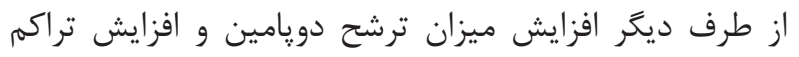

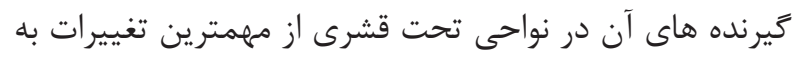
دنبال بيمارى اسكيزوفرنى مى نو باشد (1) (1).

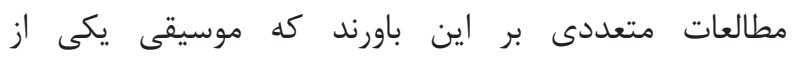

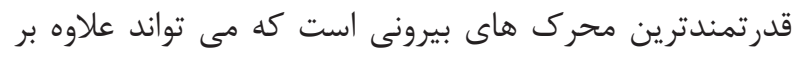

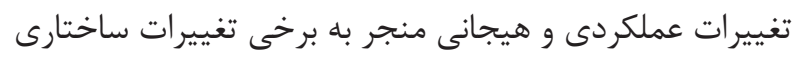

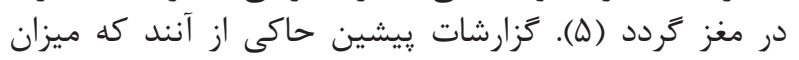

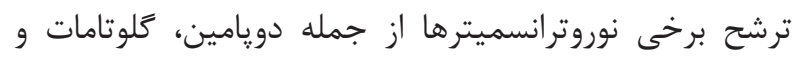

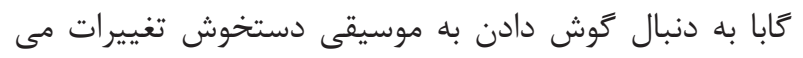

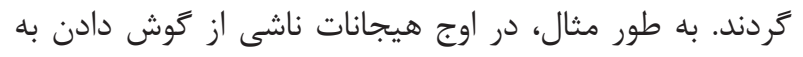

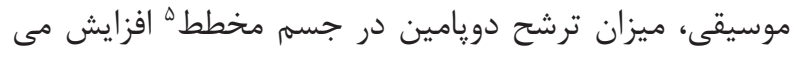

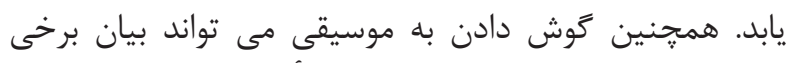

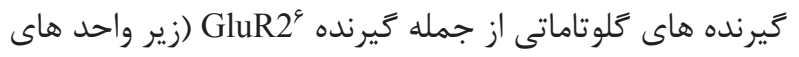

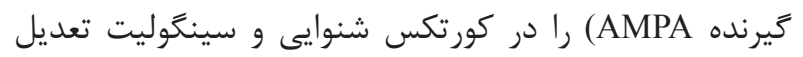

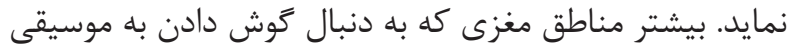

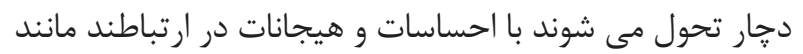

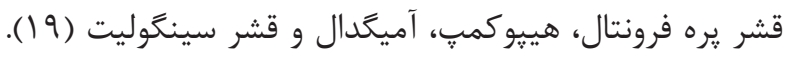

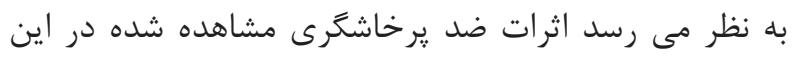

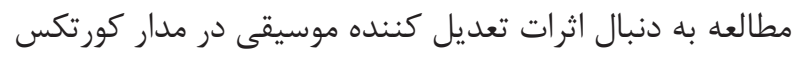

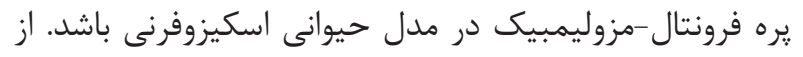

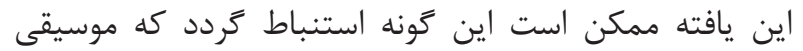

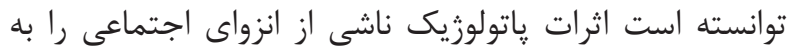

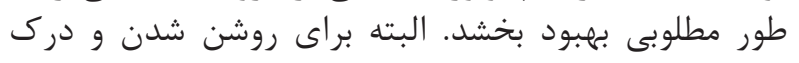

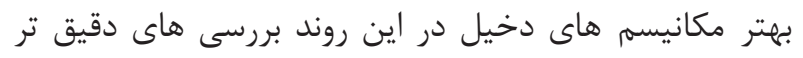

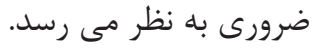

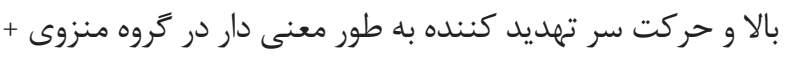

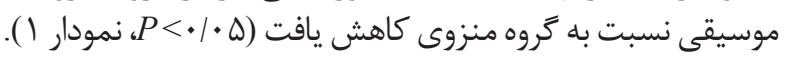

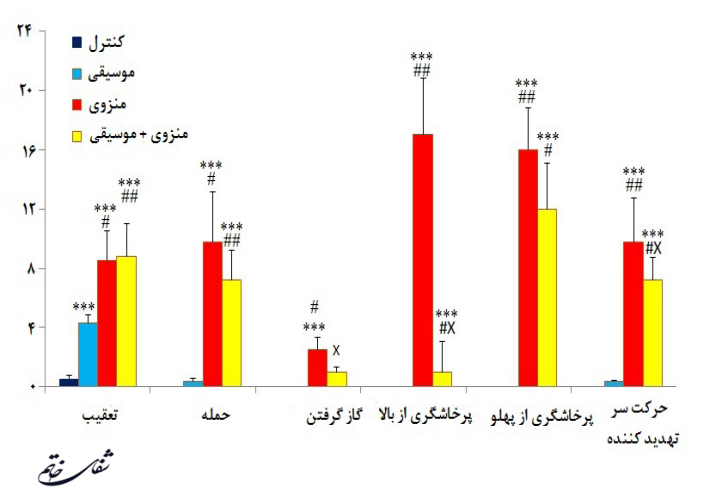

نمودار كا - مقايسه ى ميانكين نمره ى رفتارهاى يرخاشجويانه بين كروه هاى مورد آزمايش.

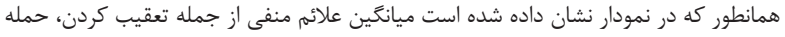

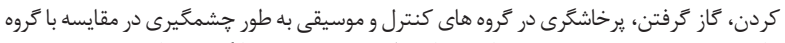

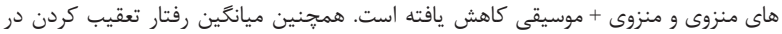

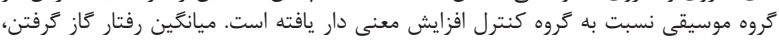

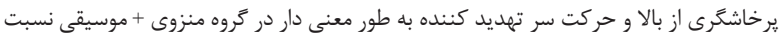

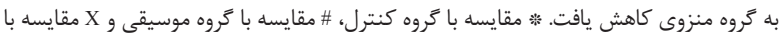

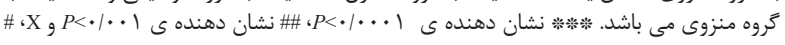

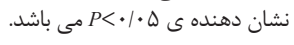

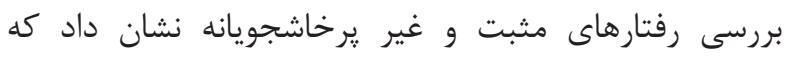

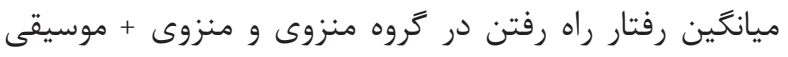

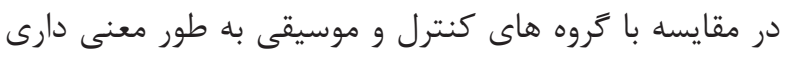

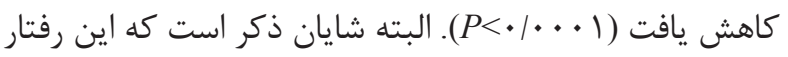

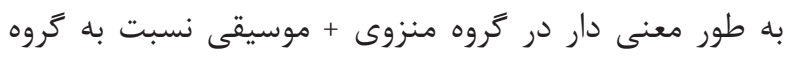

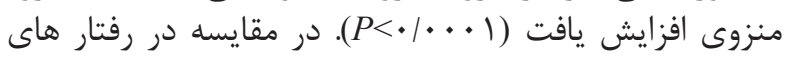

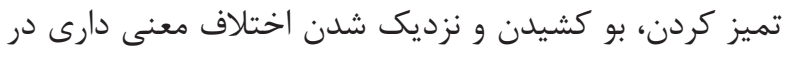

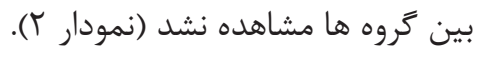

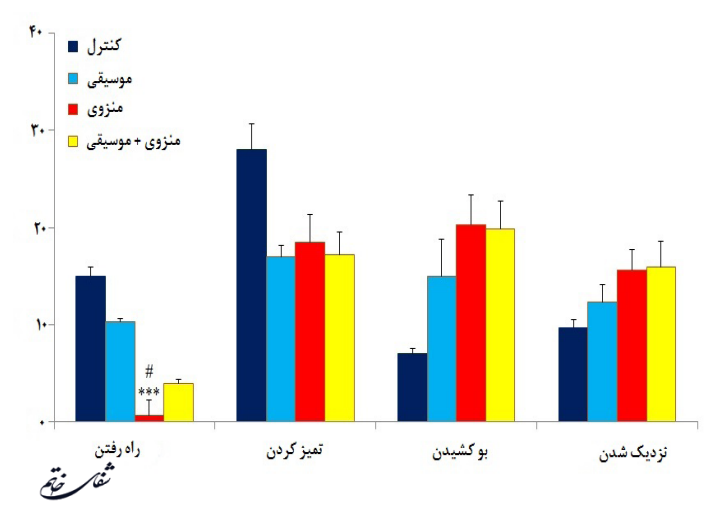

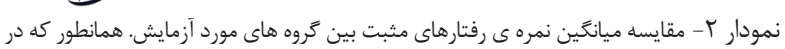

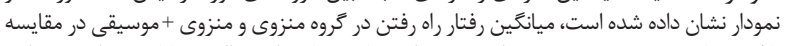

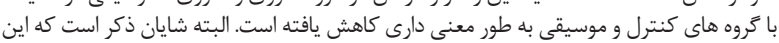

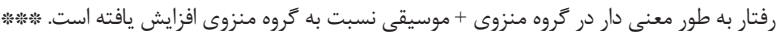

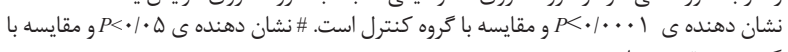
كروه موسيقى مى باشد.

\section{بحث و نتيجهَيرى}

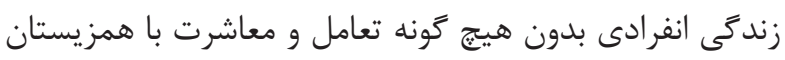

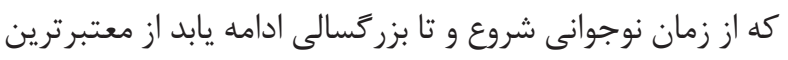

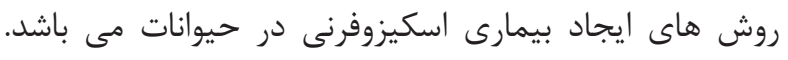

\footnotetext{
${ }^{5}$ Striatal System

${ }^{6}$ Glutamate Receptor 2
} 
1. van Os J, Rutten BP, Poulton R. Gene-environment interactions in schizophrenia: review of epidemiological findings and future directions. Schizophr Bull. 2008; 34(6): 1066-82.

2. Patkar AA, Gopalakrishnan R, Lundy A, Leone FT, Certa KM, Weinstein SP. Relationship between tobacco smoking and positive and negative symptoms in schizophrenia. J Nerv Ment Dis. 2002; 190(9): 604-10.

3. Lieberman JA, Stroup TS, McEvoy JP, Swartz MS, Rosenheck RA, Perkins DO, et al. Effectiveness of antipsychotic drugs in patients with chronic schizophrenia. N Engl J Med. 2005; 353(12): 1209-23.

4. Leucht S, Pitschel-Walz G, Abraham D, Kissling W. Efficacy and extrapyramidal side-effects of the new antipsychotics olanzapine, quetiapine, risperidone, and sertindole compared to conventional antipsychotics and placebo. A meta-analysis of randomized controlled trials. Schizophr Res. 1999; 35(1): 51-68.

5. Sacks O. The power of music. Brain. 2006; 129(10): 2528-32.

6. Baumgartner T, Lutz K, Schmidt CF, Jäncke L. The emotional power of music: how music enhances the feeling of affective pictures. Brain res. 2006; 1075(1): 151-64.

7. Lahiri N, Duncan JS. The Mozart effect: encore. Epilepsy Behav. 2007; 11(1): 152-3.

8. Dastgheib SS, Layegh P, Sadeghi R, Foroughipur M, Shoeibi A, Gorji A. The Effects of Mozart's Music on Interictal Activity in Epileptic Patients: Systematic Review and Meta-analysis of the Literature. Curr Neurol Neurosci Rep. 2014; 14(1): 1-11.

9. Newman J, Rosenbach JH, Burns KL, Latimer BC, Matocha HR, Vogt ER. An Experimental Test of "the Mozart Effect": Does Listening to His Music Improve Spatial Ability? Percept Mot Skills. 1995; 81(3f): 1379-87.

10. McKelvie P, Low J. Listening to Mozart does not improve children >s spatial ability: Final curtains for the Mozart effect. Brit J Dev Psychol. 2002; 20(2): 241-58.

11. Chikahisa S, Sei H, Morishima M, Sano A, Kitaoka $\mathrm{K}$, Nakaya Y, et al. Exposure to music in the perinatal period enhances learning performance and alters BDNF/ TrkB signaling in mice as adults. Behav brain res. 2006; 169(2): 312-9.

12. Fukui H, Toyoshima K. Music facilitate the neurogenesis, regeneration and repair of neurons. Med Hypotheses. 2008; 71(5): 765-9.

13. File SE. The use of social interaction as a method for detecting anxiolytic activity of chlordiazepoxidelike drugs. Journal of neuroscience methods. 1980; 2(3): 219-38.

14. Ellenbroek B. Schizophrenia: Animal Models. ENC Psychopharmacol. 2010: 1181-6.

15. Harrison PJ. The neuropathology of schizophrenia A critical review of the data and their interpretation. Brain. 1999; 122(4): 593-624.

16. Costa E, Davis J, Dong E, Grayson DR, Guidotti A, Tremolizzo L, et al. A GABAergic cortical deficit dominates schizophrenia pathophysiology. Crit Rev Neurobiol. 2004; 16(1\&2).

17. Nakazawa K, Zsiros V, Jiang Z, Nakao K, Kolata $\mathrm{S}$, Zhang $\mathrm{S}$, et al. GABAergic interneuron origin of schizophrenia pathophysiology. Neuropharmacology. 2012; 62(3): 1574-83.

18. Moore H, West AR, Grace AA. The regulation of forebrain dopamine transmission: relevance to the pathophysiology and psychopathology of schizophrenia. Biol Psychiatry. 1999; 46(1): 40-55.

19. Salimpoor VN, Benovoy M, Larcher K, Dagher A, Zatorre RJ. Anatomically distinct dopamine release during anticipation and experience of peak emotion to music. Nat Neurosci. 2011; 14(2): 257-62. 